Journal of Engineering and Applied Sciences 14 (Special Issue 8): 10402-10405, 2019

ISSN: 1816-949X

(C) Medwell Journals, 2019

\title{
Design an FBG Senor for Accurate Pressure Sensing
}

\author{
${ }^{1}$ Ammar F. Majeed, ${ }^{1}$ Ibraheem A. Murdas, ${ }^{2}$ Shehab A. Kadhim and ${ }^{2}$ Aseel I. Mahmood \\ ${ }^{1}$ Department of Electrical Engineering, College of Engineering, \\ University of Babylon, Hillah, Iraq \\ ${ }^{2}$ Materials Research Directorate, Ministry of Science and Technology, Baghdad, Iraq
}

\begin{abstract}
The use of photonic sensors is gaining a large acceptance as an alternative to traditional electrical and mechanical sensors for monitoring of mechanical parameters. The potential impact of these sensors is related to their good metrological properties, small size and flexibility as well as the immunity from the electromagnetic field. This FBG might be implemented as a sensor to monitor the pressure produced by strain in any real medical application such as heart rate, ECG and respiratory status. In this study, we present an implementation of the FBGs sensor in a pressure waveform observer based on the strain phenomenon. The lab experimental results for FBG sensor demonstrate high sensitivity, very little error rate and an excellent linear relationship between the pressure corresponding to Bragg wavelength/shift. The obtained results having a Mean Absolute Percentage Error (MAPE) $0.00126 \%$, sensitivity $0.1 \mathrm{Pm} / \mu$ strain and linearity factor $98.43 \%$.
\end{abstract}

$\underline{\text { Key words: Photonic sensors, mechanical sensors, mechanical parameters, wavelength, FBG, ECG }}$

\section{INTRODUCTION}

In instrumentation and sensors, optical scheme has played a significant role. Because of their feature such as cylindrical geometry, lightweight, small size, robust to environment, high in sensitivity, size compacted, possibility of remote sensing and immune to electromagnetic interference, fiber based sensors offer several important benefits over typical electronic sensors and won the special attraction (Malla et al., 2008).

In addition to aforementioned features, optical fiber sensors have specific properties such as low manufacturing cost, miniature, flexible structures, capability of distributive and multi-parameter sensing on a single fiber (Fidanboylu and Efendioglu, 2009) and chemically inert. They become able to be utilized in the process of chemic and biomedical instrumentation.

In a refractive index in an optic fiber, a Fiber Bragg Grating (FBG) is a periodic change and therefore, it is implemented as a wavelength specific selector (Andreas and Kalli, 1999). Because of the selective property in the FBGs, it becomes an important tool used in telecommunication, sensors and other optics applications.

FBG sensors are able to be implemented in different applications of sensing to compute physical quantities such as strain, temperature, pressure, ultrasound and high magnetic field, force (Chan et al., 2006). In recent years, the implementation of FBG sensors was reported in humidity sensing, torque reveal on acceleration sensing, rotating shafts and ultrasonic imaging of seismic physical models (Juca and Santos, 2017). FBGs have been implemented to other different areas such as vibrations of mechanical. The mechanical vibrations contain non-destructive testing, smart materials and structural health controlling (Wild and Hinckley, 2010). Fiber Bragg Gratings (FBGs) can be effectively utilized in an extensive variety of biomedical applications, one of them is tumor detection (Moosa, 2017).

Typically the Bragg gratings reflect specific wavelength range that satisfies the all other wavelengths. So, FBGs become a great importance to be utilized as smart sensors in different applications such as medical applications to monitor patient health care, strain and temperature measurements (Murdas and Jabbar, 2018).

\section{MATERIALS AND METHODS}

Problem formulation: To exhibit the ability of FBG as accurate pressure sensor to be utilized in medical applications such as pulse rate, heartbeat and ECG. Where the FBG has good pressure sensitivity, good accuracy of static and dynamic measuring, immunity against electrical and magnetic interference and miniature size compared to the traditional electric sensors.

System model: Coupled-mode theory (Sipe et al., 1994) is a good tool for obtaining quantitative information about the diffraction efficiency and spectral dependence of fiber gratings. It is a straightforward and intuitive method to accurately model the optical properties of most fiber gratings of interest. Full details could be found in (Kashyap, 2009). In this work the utilized mathematical model is convenient to be implemented in the FBG pressure or strain sensor as follow:

Corresponding Author: Ammar F. Majeed, Department of Electrical Engineering, College of Engineering, University of Babylon, Hillah, Iraq 
According to the Bragg condition, the center period of grating it is easy to be determine (Kashyap, 2009) as follows equation:

$$
\lambda_{\mathrm{B}}=2 \mathrm{n}_{\mathrm{eff}} \Lambda
$$

where, $\lambda_{B}, n_{\text {eff }}$ and $\Lambda$ denotes the Bragg wavelength, effective refractive index and gratings period, respectively. The change in the wavelength and power of FBG depends on the distance between the Bragg planes. If this distance changed due to any external influence the wavelength and power will be changed. A specific mechanism such as MANGANA utilized to generate axial strain on the sensor to exhibit the realization and characterization of FBG as a pressure sensor. In this case $\Delta \mathrm{T}$ equals to zero then the Bragg wavelength shifts become as a function of the physical elongation and the Pockels coefficients. According to Eq. 2 the Bragg wavelength under strain effects is described by:

$$
\begin{gathered}
\frac{\Delta \lambda_{\mathrm{B}}}{\lambda_{\mathrm{B}}}=\mathrm{k} \\
\Delta \lambda_{\mathrm{B}}=\lambda_{\mathrm{B}}[1-\mathrm{P}] \epsilon
\end{gathered}
$$

Where:

$$
=\frac{\mathrm{n}_{\text {eff }}^{2}}{2}\left[P_{12}-\left(P_{11}+P_{12}\right)\right]
$$

where is the Poisson ratio is an effective strain optic term. Since, is equal to 0.22 and is worth 0.78 . Therefore, the strain is given by Kreuzer (2006), Maier et al. (2004):

$$
=\frac{\Delta \lambda_{\mathrm{B}}}{\lambda_{\mathrm{B}}} \frac{1}{0.78}
$$

The strain represents the percentage of the change in FBG length to the original length as illustrated in the following equation (Kisala, 2012):

$$
=\frac{\Delta \mathrm{L}}{\mathrm{L}}
$$

Then the change in FBG length is obtained by:

$$
\Delta \mathrm{L}=\frac{\Delta \lambda_{\mathrm{B}}}{\lambda_{\mathrm{B}}} \frac{\mathrm{L}}{0.78}
$$

Proposed framework: The principle work of FBG pressure sensor is explained by changes in Bragg wavelength shift in accordance with the strain waveforms acquired in a real-time. In order to design and implemented an accurate $\mathrm{FBG}$ pressure sensor, a realistic physical model has been created to act the pressure rules at the real sensor location.

To implement FBG sensor for measuring strain, we create a realistic physical model for simulating the strain

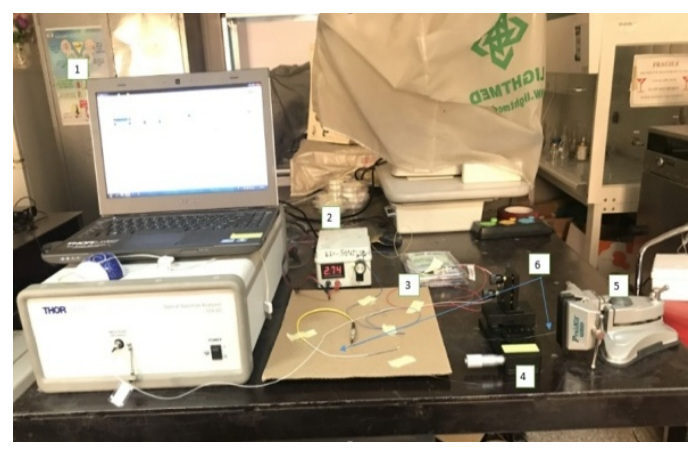

Fig. 1: Experimental setup of FBG pressure sensor, (1) OSA, (2) Laser source, (3) Optical circulator, (4) MMSM, (5) Fixation device and (6) FBG sensor

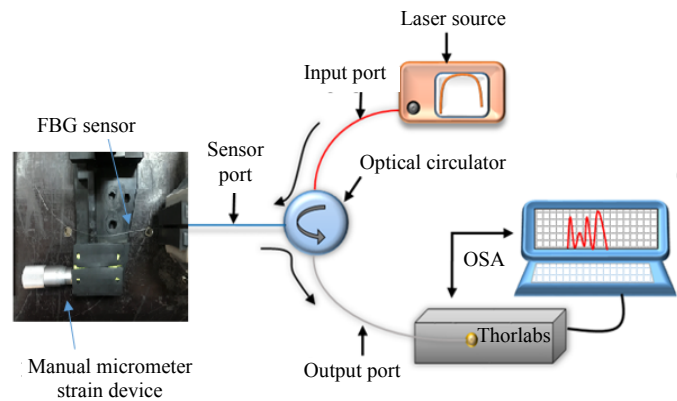

Fig. 2: Schematic diagram of FBG pressure sensor

produced by Manual Micrometer Strain Maker device (MMSM) that produces an axial strain in the range of 60-300 $\mu$ strain. The schematic diagram and experimental setup of the FBG pressure sensor are shown in Fig. 1 and 2 , respectively.

This model consists of MMSM devise to build a strain pulse waveform. The fixation of sensing area of the FBG (Bragg grating zone) from both ends is done by MMSM device and another fixing machine. Fiber-Coupled Laser diode Source (LPS-FC) having wavelength $1550 \mathrm{~nm}$ and optical power $1.5 \mathrm{~mW}$ had been connected to the input port of polarization independent SMF optical circulator. The sensing port (the second arm of optical circulator) had been launched to the input port of FBG (having wavelength $1550 \pm 0.3 \mathrm{~nm}$ ). The end side of the FBG sensor was located on the MMSM device.

The reflected light from FBG was propagated through the detection port (the third arm of optical circulator) which was launched to an optical spectrum analyzer OSA (Model Thorlabs 203 with resolution $0.01 \mathrm{~nm}$ ). The function of the optical circulator is to control the direction of the signal. When the light signal enters the circulator through the first arm the circulator directs the signal to the FBG through the second arm and prevents it from through the third arm. While the signal is reflected from the FBG, it will be redirected to the OSA through the third arm. 


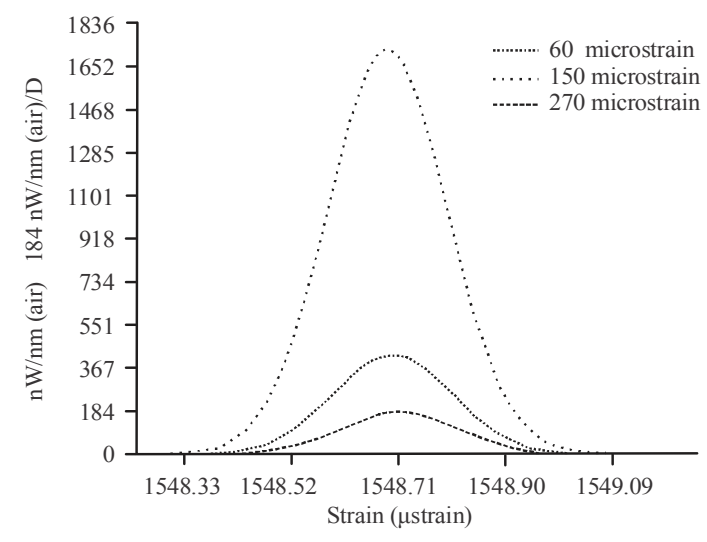

Fig. 3: The strain effect on Bragg wavelength

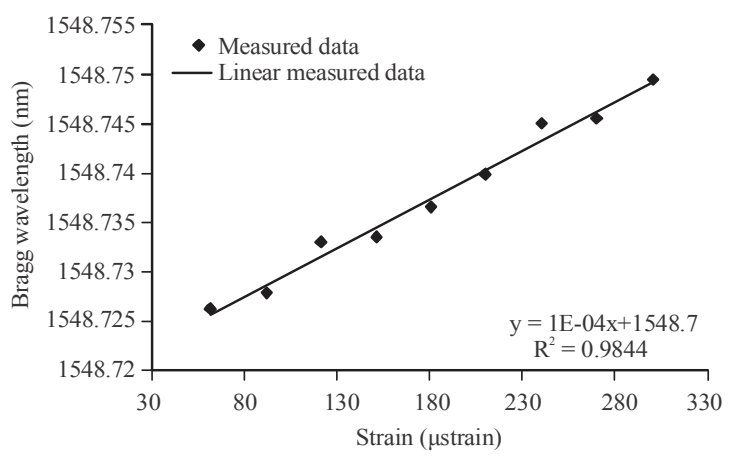

Fig. 4: The linearity of FBG pressure sensor

\section{RESULTS AND DISCUSSION}

In this section, the outcomes obtained from the experimental activities are presented in details. In this experiment, we proved that the utilized FBG could be implemented as an optical pressure sensor through a realistic physical model as aforementioned in section IV.

The shift in reflected Bragg wavelength and optical power obtained from waveforms (which is pulse waves caused by the pressure obtained by MMSM) are shown in Fig. 3. The relationship between Bragg wavelengths with the applied pressure is shown in Fig. 4.

As shown in these figures the relationship between the pressure and wavelength is a proportional relationship. Incensement in pressure causes an increment in wavelength shift which is due to the change in distance between periodic planes produced by the axial strain. Output power is one of the variables affected by the strain and it was measured with identical conditions. A linear graph presented in Fig. 4 proofs the theory of FBG which is when the pressure is increased the Bragg wavelength is increased.

The results of accuracy parameters obtained from the FBG pressure sensor are shown in Table 1 below where it is shows high sensitivity, low percentage error (MAPE) and excellent linearity.
Table 1: The accuracy parameters of FBG pressure sensor

\begin{tabular}{ll}
\hline Parameters & Values \\
\hline Correlation factor $\left(\mathrm{R}^{2}\right)$ & 0.9844 \\
Sensitivity & $0.1 \mathrm{pm} / \mu \mathrm{strain}$ \\
Mean Absolutely Division (MAD) & $0.01944 \mathrm{~nm}$ \\
Mean Square Error (MSE) & $0.00379 \mathrm{~nm}^{2}$ \\
Mean Absolutely Percentage Error (MAPE) & $0.00126 \%$ \\
\hline
\end{tabular}

In principle the results were consistent with the results of scientific publications according to the compared related works. Pei et al. (2018), Zhang et al. (2019), Elgaud et al. (2016). The results evidence that the bare fiber Bragg grating has higher efficiency, lower MAPE and higher sensitivity as the most previous literature of the related work.

\section{CONCLUSION}

In this research, a novel system to realize and characterize the FBG pressure sensor is implemented. The system evident that the accuracy of the obtained results are consistent with the previous related works. It could be used for real applications. The FBG sensor plays a very important role in this system which is also has a convenient specifications, especially in low cost, fast response and measurement continuity. The submitted FBG sensors show high sensitivity about $0.1 \mathrm{Pm} / \mu$ strain, also these sensors exhibit a small error rate described in MSE $0.00379 \mathrm{~nm}^{2}$, MAD $0.01944 \mathrm{~nm}$ and MAPE $0.00126 \%$. The FBG demonstrated excellent linearity between applied physics and shifting in Bragg wavelength $(98.44 \%)$.

\section{REFERENCES}

Andreas, O. and K. Kalli, 1999. Fiber Bragg Gratings: Fundamentals and Applications in Telecommunications and Sensing. 2nd Edn., Artech House, Norwood, MA.

Chan, T.H.T., L. Yu, H.Y. Tam, Y.Q. Ni, S.Y. Liu, W.H. Chung and L.K. Cheng, 2006. Fiber Bragg grating sensors for structural health monitoring of Tsing Ma bridge: Background and experimental observation. Eng. Struct., 28: 648-659.

Elgaud, M.M., M.S.D. Zan, A.A.G. Abushagur and A.A.A. Bakar, 2016. Analysis of independent strain-temperature fiber Bragg grating sensing technique using OptiSystem and OptiGrating. Proceedings of the 2016 IEEE 6th International Conference on Photonics (ICP), March 14-16, 2016, IEEE, Kuching, Malaysia, pp: 1-3.

Fidanboylu, K.A. and H.S. Efendioglu, 2009. Fiber optic sensors and their applications. Proceedings of the 5 th International Symposium on Advanced Technologies (IATS'09), May 13-15, 2009, Karabuk, Turkey, pp: 1-6. 
Juca, M.A. and A.B.D. Santos, 2017. Fiber Bragg grating interrogation using FBG filters and artificial neural network. Proceedings of the 2017 SBMO/IEEE MTT-S International Conference on Microwave and Optoelectronics (IMOC), August 27-30, 2017, IEEE, Aguas de Lindoia, Brazil, pp: 1-4.

Kashyap, R., 2009. Fiber Bragg Gratings. 2nd Edn., Academic Press, Cambridge, Massachusetts, USA., ISBN: 9780123725790, Pages: 632.

Kisala, P., 2012. Optoelectronic sensor for simultaneous and independent temperature and elongation measurem ent using Bragg gratings. Electrotechnical Rev., 88: 343-346.

Kreuzer, M., 2006. Strain measurement with fiber Bragg grating sensors. Hottinger Baldwin Messtechnik GmbH, Darmstadt, Germany. http://www.ae.metu. edu.tr/seminar/strain-gage/FBG.pdf

Maier, R.R.J., W.N. MacPherson, J.S. Barton, J.D.C. Jones, S. McCulloch and G. Burnell, 2004. Temperature dependence of the stress response of fibre Bragg gratings source. Meas. Sci. Technol., 15: 1601-1606.

Malla, R.B., A. Sen and N. Garrick, 2008. A special fiber optic sensor for measuring wheel loads of vehicles on highways. Sens., 8: 2551-2568.
Moosa, A.A., 2017. Fiber Bragg grating in biomedical application. Al. Nahrain J. Eng. Sci., 20: 636-640.

Murdas, I.A. and A.N. Jabbar, 2018. Online healthcare monitor system based on optical wireless and FBG sensors. Int. J. Eng. Technol., 7: 100-106.

Pei, Y., T. Liao, J. Xu, H. Lin and T. Ning, 2018. FBG strain sensor applied in harsh environment of aerospace. Proceedings of the 2018 IEEE 3rd Conference on Optoelectronics Global (OGC), September 4-7, 2018, IEEE, Shenzhen, China, pp: 81-84.

Sipe, J.E., L. Poladian and C.M.D. Sterke, 1994. Propagation through nonuniform grating structures. J. Opt. Soc. Am. A., 11: 1307-1320.

Wild, G. and S. Hinckley, 2010. Optical fibre Bragg gratings for acoustic sensors. Proceedings of the 20th International Congress on Acoustics (ICA 2010), August 23-27, 2010, Sydney, Australia, pp: 1-7.

Zhang, W., W. Zhuang, M. Dong, L. Zhu and F. Meng, 2019. Dual-parameter optical fiber sensor for temperature and pressure discrimination featuring cascaded tapered-FBG and ball-EFPI. IEEE. Sens. J., 14: 5645-5652. 